\title{
The Role of Insula in Physical Activity Moderate the Association Between Problematic Mobile Phone Use and Emotional Symptoms in Late Adolescents
}

\author{
Liwei Zou \\ Anhui Medical University \\ Xiaoyan Wu \\ Anhui Medical University \\ Shuman Tao \\ the Second Hospital of Anhui Medical University \\ Yajuan Yang \\ Anhui Medical University \\ Qingjun Zhang \\ Ping An Healthcare Diagnostics Center \\ Xuedong Hong \\ Ping An Healthcarre Diagnostics Center \\ Honglv Xu \\ Anhui Medical University \\ Yang Xie \\ Anhui Medical University \\ Tingting Li \\ Anhui Medical University \\ Suisheng Zheng \\ Ping An Healthcare Diagnostics Center \\ Fangbiao Tao ( $\square$ taofangbiao@126.com) \\ Anhui Medical University https://orcid.org/0000-0003-4807-9670
}

Research

Keywords: smartphone addiction, physical activity, moderating effect, mental health, MRI

Posted Date: May 21st, 2020

DOI: https://doi.org/10.21203/rs.3.rs-30314/v1

License: @) (i) This work is licensed under a Creative Commons Attribution 4.0 International License. Read Full License 


\section{Abstract}

Background: Problematic mobile phone use (PMPU) and psychopathological symptoms are great public health concerns in adolescents. Previous studies have shown the associations between PMPU and emotional symptoms, but few studies to explore physical activity (PA) and the neural structures correlated with PA which moderate the relationship between PMPU and emotional symptoms. The aim of current study is to examine the moderating effect of PA and neural basis of such moderating effect on the relation between PMPU and emotional symptoms.

Methods: A total of 251 college students underwent magnetic resonance imaging scanning. PMPU, PA and emotional symptom were assessed by self-rating questionnaire for adolescent problematic mobile phone use (SQAPMPU), international physical activity questionnaire (IPAQ-C), and depression anxiety stress scale-21 (DASS-21), respectively. A multiple regression model was performed to detect brain structure-gray matter volume associated with PA by voxel-based morphometry method. Moderating analysis was conducted using PROCESS macro in the SPSS software.

Results: PA has significantly moderate effect on the association between PMPU with depressive $(\beta=0.301, p<0.05)$, anxiety $(\beta=0.328, p<0.05)$ and stress $(\beta=0.343, p<0.05)$ symptoms. PA was correlated to the GMV of the right fusiform gyrus, the left precuneus, the left insula, and the left triangular part of inferior frontal gyrus. The relation between PMPU and depressive symptom was moderated by greater GMV of left insula.

Conclusions: The findings indicate that high levels of PA can reducing the association between PMPU and emotional symptoms, and further find the GMV of left insula which correlated with PA may play a key role on the relationship between PMPU and depressive symptom. The intervention programs of emotional symptoms and insula-based deep brain stimulation is discussed as future study.

\section{Background}

In current young generation, it experiences rapid developments of emerging technologies, especially portable electronic devices-mobile phone. Mobile phone has become an indispensable part of our daily life due to accessing the internet. It is reported that the prevalence of mobile phone use is $90.8 \%$ globally from Globalwebindex (https://www.globalwebindex.com/). In China, there were 846.8 million mobile phone users by the end of August in 2019, 26.0\% of whom were students (1). Thus, the use of mobile phone is attracting a growing concern and is drawing research attention.

Along with many advantages from mobile phone with access to information and communication fast, many studies discussed the potential negative consequences of mobile phone overuse in recent years. Problematic mobile phone use (PMPU) has also been termed mobile phone dependence and mobile phone addiction (2), which defined as excessive use with features of craving, tolerance and dependence that resulted adverse health and functional consequences (3-5).

Recent evidence indicates that PMPU has been associated with bodily pain, sleep problems and mental health (6-8). Multiple studies have documented significant association between PMPU and mental disorders in adolescents. For example, a longitudinal study by Lapierre et al (9) found the association between PMPU and depression in late adolescents. Similarly, studies of Korean, Serbia, Italy and American adolescents reported that intensity of mobile phone use were significantly associated with anxiety and depression (10-12). Moreover, a systematic review included ten studies found most support for relationships between PMPU and depression and anxiety severity in different sample of adolescents and adults (13).

Nowadays, it has attracted more attention on physical activity (PA) as a treatment for mental health disorders (14). In a large cross-sectional study (15), individuals who exercised had better mental health than individuals who did not exercise. Moreover, individuals with regular leisure-time exercise were less develop depression from a large cohort study (16). In addition, meta-analysis from randomized clinical trials had reported that PA is associated with reduction of depressive symptom and supported that PA is a treatment for depression (17).

Although such researches indicate that PA can reduce emotional symptoms, the mechanism of protective role of PA for mental health disorder was still unclear. Several recent studies have found relationship between PA and brain morphology $(18,19)$. We therefore hypothesize that a relationship between PMPU and emotional symptoms are moderated by gray matter volume (GMV) of PA-related brain regions.

The aim of present study is (a) to replicate the relationship between PMPU and emotional symptoms, (b) to examine the moderate effect of PA and (c) to detect neural structures correlated with PA which moderate such a relationship.

\section{Materials And Methods}

\subsection{Participants}

This cross-sectional survey was performed among freshmen from 2 school and 5 different majors at one University in Hefei, Anhui Province from April 2019 to June 2019. Data were collected from 574 participants and of 268 participants obtained MRI scan in this study. Of the 268 college students, a total of 17 were excluded due to incidental finding $(n=1)$ and missing information on physical activity $(n=16)$, the sample of present 
study included 251 college students (mean age $19.01 \pm 0.85$ years, $20.72 \%$ males). A flow chart shows the exclusion of data in Fig. 1 . The study approved by Ethics Committee of Anhui Medical University and all participants were written informed consent.

\subsection{Measures}

\subsubsection{Assessment of PMPU}

The Self-rating Questionnaire for Adolescent Problematic Mobile Phone Use (SQAPMPU) (20) was a 13-item measure which included 3 dimensions: withdrawal symptoms, craving, and physical and mental health status. Each item was rated on a 5-point Likert scale (Not true at all $=1$, Slightly true $=2$, Moderately true $=3$, Strongly true $=4$, Extremely true $=5$ ), so that the total score was possible ranged from 13 to 65 . Higher scores indicated higher levels of problematic mobile phone use, and the Cronbach's alpha coefficient of the scale was 0.89.

\subsubsection{Assessment of PA}

Physical activity was assessed by the 7-item Chinese version of the International Physical Activity Questionnaire (IPAQ-C) (21), with classified 3 types of PA: walking, moderate physical activity (MPA, e.g. carrying a light load, swimming, and cycling) and vigorous physical activity (VPA, e.g. carrying or lifting heavy loads, digging and running). Participants were obtained the frequency (days per week) and duration (minutes per day) of each activity during the last 7 days. The amount of PA was processed into metabolic equivalent (MET) min/week using MET values for walking (3.3), MPA (4.0), and VPA (8.0). The MET min/week of each activity was calculated by its value of MET $\times$ frequency (day/week) $\times$ duration (min/day). The total PA was a sum of total (walking + MPA + VPA) MET min/week.

\subsubsection{Assessment of Emotional symptoms}

The Chinese version of the Depression Anxiety Stress Scale-21 (DASS-21) was assessed emotional symptoms in college students. The DASS-21 was consisted of 21 items for measuring depression, anxiety, and stress symptoms. Each subscale was measured by 7 items with 4 response options from 0 (not at all) to 3 (very much or most of the time). The total score was possible ranged from 0 to 63 , and higher scores indicated severe emotional symptoms. The depression, anxiety, and stress symptoms were defined by 9,7 , and 14 scores in each subscale (22). The Cronbach's alpha coefficient of our study were 0.85 for depression, 0.78 for anxiety and 0.84 for stress.

\subsection{MRI}

\subsubsection{Image acquisition}

All MRI data were acquired with 3.0 T Philips Ingenia CX scanner (Philips, Best, Netherlands) in the Ping An Healthcare Diagnostics Center (Hefei, Anhui, China). Polyurethane foam pads and earmuffs were used to minimize head motion and reduce scanner noise during scanning. The 3D high resolution T1-weighted structural images were acquired with fast field echo (FFE) technique by following parameters: echo time $=3.2$ ms, repetition time $=7.1 \mathrm{~ms}$, field of view $=256 \times 256 \mathrm{~mm}^{2}$, slice thickness $=1 \mathrm{~mm}$, voxel size $1 \times 1 \times 1 \mathrm{~mm}^{3}$, number of slices $=180$. The acquisition time was 5 minutes and 5 seconds.

\subsubsection{Data preprocessing}

VBM of 3D high resolution T1-weighted structural images were performed using the Computational Anatomy Toolbox (CAT, http://dbm.neuro.unijena.de/cat/) and Statistical Parametric Mapping (SPM12, http://www.fil.ion.ucl.ac.uk/spm/). The main process of VBM included the segmentation of structural images into gray matter (GM), white matter (WM) and cerebrospinal fluid (CSF), normalization by diffeomorphic anatomical registration using exponentiated lie algebra (DARTEL) method, and smoothing of GMV segments with a 6 mm full width at half maximum (FWHM) isotropic Gaussian kernel.

\subsection{Statistical analysis}

Data analysis was conducted in SPSS version 23.0 (SPSS, Chicago, IL, USA). The descriptive statistics were used mean (SD) and median for continuous variables, and frequencies and percentages for categorical variables. The statistical significance was set at $p<0.05$.

To address our hypotheses (see Fig. 2), we conducted the following analysis steps.

First, linear regression analysis was conducted to explore association between PMPU and emotional symptoms including depression, anxiety and stress scores as outcomes, and SQAPMPU scores as predictor (step 1).

Second, we examined whether PA moderates the association between PMPU and emotional symptoms. Before to moderation analyses, PA from total MET were log-10 transformed, PMPU and PA were mean-centered to reduce multicollinearity (23). A moderation analysis was conducted with PMPU as independent variable, PA as moderator, and emotional symptoms as dependent variable by SPSS PROCESS macro, version 3.0 (model 1), developed by Hayes (step 2) (24).

Third, we used voxel-based multiple regression analyses (based on general linear model) in SPM12 with voxel-wise GMV value as dependent variable and PA as a covariate of interest to investigate the potential association between PA and brain structure. Moreover, gender as nuisance 
covariance to control its effect on brain structure (step 3). The results were set the significant value at $p<0.05$ (height threshold of $p<0.001$ ) with family-wise error (FWE) correction for multiple comparisons.

Finally, we aimed to assess whether PA-related GMV moderates the association between PMPU and emotional symptoms. Therefore, we extracted GMV from brain regions that strongly $\left(P_{\mathrm{FWE}}<0.05\right)$ associated with PA. The procedure as same as above, except the extracted GMV that correlated with PA as moderator. Simple slopes to show the association between PMPU and emotional symptoms at low $(M-1 S D)$ and high $(M+1 S D)$ levels of the moderator.

\section{Results}

There were $13.1 \%, 23.1 \%$ and $7.2 \%$ of participants who had depressive, anxiety and stress symptom in present study, respectively. Table 1 presented the demographic characteristics in different emotional symptoms.

Table 1

Demographic characteristics of participants.

\begin{tabular}{|c|c|c|c|c|c|c|c|c|c|c|}
\hline \multirow[t]{2}{*}{ Variables } & \multirow[t]{2}{*}{$\mathbf{N}$} & \multicolumn{3}{|c|}{ Depression symptoms } & \multicolumn{3}{|c|}{$\begin{array}{l}\text { Anxiety } \\
\text { symptoms }\end{array}$} & \multicolumn{3}{|c|}{$\begin{array}{l}\text { Stress } \\
\text { symptoms }\end{array}$} \\
\hline & & $\%$ & $x^{2}$ & $P$ & $\%$ & $x^{2}$ & $P$ & $\%$ & $\chi^{2} /$ Fishe's test & $P$ \\
\hline Gender & & & 0.01 & 0.940 & & 0.14 & 0.707 & & - & 0.771 \\
\hline Male & 52 & 13.5 & & & 21.2 & & & 7.7 & & \\
\hline Female & 199 & 13.1 & & & 23.6 & & & 7.0 & & \\
\hline Residential Area & & & 0.16 & 0.687 & & 1.13 & 0.288 & & 1.41 & 0.235 \\
\hline Rural & 145 & 12.4 & & & 20.1 & & & 5.5 & & \\
\hline Urban & 106 & 14.2 & & & 26.4 & & & 9.4 & & \\
\hline Any siblings & & & 0.01 & 0.917 & & 0.22 & 0.640 & & 0.11 & 0.742 \\
\hline Yes & 55 & 12.7 & & & 25.5 & & & 9.1 & & \\
\hline No & 196 & 13.3 & & & 22.4 & & & 6.6 & & \\
\hline Perceived family income & & & 4.66 & 0.098 & & 10.22 & 0.006 & & 0.45 & 0.905 \\
\hline Low & 49 & 16.3 & & & 30.6 & & & 8.2 & & \\
\hline Medium & 189 & 11.1 & & & 19.0 & & & 6.9 & & \\
\hline High & 13 & 30.8 & & & 53.8 & & & 7.7 & & \\
\hline Academic performance & & & 0.72 & 0.699 & & 0.03 & 0.985 & & 3.79 & 0.134 \\
\hline Poor & 45 & 11.1 & & & 22.2 & & & 13.3 & & \\
\hline Medium & 158 & 12.7 & & & 23.4 & & & 5.1 & & \\
\hline Good & 48 & 16.7 & & & 22.9 & & & 8.3 & & \\
\hline Father's educational level & & & 4.61 & 0.100 & & 1.93 & 0.381 & & 0.30 & 0.928 \\
\hline Primary school or lower & 49 & 22.4 & & & 30.6 & & & 8.2 & & \\
\hline Middle school & 174 & 10.9 & & & 21.3 & & & 6.9 & & \\
\hline College or above & 28 & 10.7 & & & 21.4 & & & 7.1 & & \\
\hline Mother's educational level & & & 0.10 & 0.953 & & 0.21 & 0.899 & & 0.07 & 0.966 \\
\hline Primary school or lower & 119 & 12.6 & & & 21.8 & & & 6.7 & & \\
\hline Middle school & 119 & 13.4 & & & 24.4 & & & 7.6 & & \\
\hline College or above & 13 & 15.4 & & & 23.1 & & & 7.7 & & \\
\hline
\end{tabular}

\subsection{Association of PMPU with emotional symptoms}



89.66, $\left.p<0.05, \mathrm{R}^{2}=0.27\right)$ and stress symptoms $\left(\mathrm{F}[1,249]=73.26, p<0.05, \mathrm{R}^{2}=0.23\right)$.

\subsection{Association of PA with GMV}

The total PA was positively correlated to several brain regions including the right fusiform gyrus (FFG), the left precuneus (PCUN), the left insula (INS), and the left triangular part of inferior frontal gyrus (IFGtriang) (for all results were $P_{\mathrm{FWE}}<0.05$, Table 2, Fig. 3). No negative correlation results were observed.

Table 2

Brain regions in which GMV were significantly correlated with total PA.

\begin{tabular}{|c|c|c|c|c|c|c|}
\hline \multirow[t]{2}{*}{ Cluster } & \multirow[t]{2}{*}{ Region } & \multirow[t]{2}{*}{ Cluster size } & \multicolumn{3}{|c|}{ Peak MNI(mm) } & \multirow[t]{2}{*}{ Peak T value } \\
\hline & & & $\mathbf{x}$ & $\mathbf{y}$ & $\mathbf{z}$ & \\
\hline 1 & FFG_R & 40 & 19.5 & -1.5 & -37.5 & 4.30 \\
\hline 2 & PCUN_L & 43 & -4.5 & -54 & 10.5 & 4.09 \\
\hline 3 & INS_L & 47 & -34.5 & 1.5 & 10.5 & 4.08 \\
\hline 4 & IFGtriang _L & 135 & -31.5 & 31.5 & 1.5 & 5.53 \\
\hline
\end{tabular}

\subsection{Association of PMPU with emotional symptoms were moderated by PA and PA- related GMV}

For moderation analysis, we tested whether the association between PMPU and emotional symptoms was reduced by introducing PA and GMV of PA-related brain regions. In result, we found the significantly moderate effect of PA on the association between PMPU with depressive ( $\beta=0.301$, $\left.\Delta \mathrm{R}^{2}=0.032, p<0.05\right)$, anxiety $\left(\beta=0.328, \Delta \mathrm{R}^{2}=0.017, p<0.05\right)$ and stress $\left(\beta=0.343, \Delta \mathrm{R}^{2}=0.024, p<0.05\right)$ symptoms (Table 3$)$. On the other hand for GMV of PA-related brain regions, we found left INS $\left(\beta=0.327, \Delta \mathrm{R}^{2}=0.012, p<0.05\right)$ had significantly moderate the association between PMPU and depressive symptom (Table 3, Fig. 4), and did not found the moderate effect of any other PA-related brain regions on anxiety or stress symptom. In addition, this brain region was still had moderated effect on the relationship between PMPU and depressive symptom by controlling some sociodemographic data (supplement). 
Table 3

Results from the moderated regression analysis predicting emotional symptoms.

\begin{tabular}{|c|c|c|c|c|c|c|c|c|c|c|c|c|c|}
\hline & \multirow[t]{2}{*}{ Predictors } & \multicolumn{4}{|c|}{ Depression } & \multicolumn{4}{|c|}{ Anxiety } & \multicolumn{4}{|l|}{ Stress } \\
\hline & & $\beta$ & $\mathrm{t}$ & $\Delta \mathrm{R}^{2}$ & $\mathrm{~F}$ & $\beta$ & $\mathrm{t}$ & $\Delta \mathrm{R}^{2}$ & $\mathrm{~F}$ & $\beta$ & $\mathrm{t}$ & $\Delta \mathrm{R}^{2}$ & $\mathrm{~F}$ \\
\hline \multirow[t]{3}{*}{1} & PMPU & 0.301 & $8.100^{\star *}$ & 0.032 & $10.682^{*}$ & 0.328 & $9.047^{\star \star}$ & 0.017 & $5.943^{*}$ & 0.343 & $8.124^{\star \star}$ & 0.024 & $8.066^{*}$ \\
\hline & PA & -1.810 & $-2.092^{*}$ & & & -1.939 & $-2.294^{*}$ & & & -2.478 & $-2.523^{\star}$ & & \\
\hline & PMPU × PA & -0.359 & $-3.268^{\star \star}$ & & & -0.262 & $-2.438^{\star}$ & & & -0.354 & $-2.840^{\star \star}$ & & \\
\hline \multirow[t]{3}{*}{2} & PMPU & 0.329 & $8.661^{\star \star}$ & 0.006 & 1.777 & 0.350 & $9.444^{\star \star}$ & 0.000 & 0.107 & 0.378 & $8.794^{\star \star}$ & 0.002 & 0.668 \\
\hline & FFG_R & 3.922 & 1.313 & & & 2.062 & 0.707 & & & 6.268 & 1.853 & & \\
\hline & $\begin{array}{l}\text { PMPU x } \\
\text { FFG_R }\end{array}$ & 0.497 & 1.333 & & & 0.119 & 0.328 & & & 0.345 & 0.817 & & \\
\hline \multirow[t]{3}{*}{3} & PMPU & 0.320 & $8.498^{\star \star}$ & 0.003 & 0.802 & 0.345 & $9.407^{\star \star}$ & 0.000 & 0.012 & 0.365 & $8.536^{\star \star}$ & 0.001 & 0.279 \\
\hline & PCUN_L & 1.669 & 0.573 & & & -1.440 & -0.509 & & & 0.999 & 0.302 & & \\
\hline & $\begin{array}{l}\text { PMPU } \times \\
\text { PCUN_L }\end{array}$ & 0.327 & 0.896 & & & 0.039 & 0.109 & & & 0.219 & 0.528 & & \\
\hline \multirow[t]{3}{*}{4} & PMPU & 0.327 & $8.752^{\star \star}$ & 0.012 & $3.890^{*}$ & 0.352 & $9.662^{* *}$ & 0.005 & 1.849 & 0.369 & $8.604^{\star *}$ & 0.001 & 0.417 \\
\hline & INS_L & -5.832 & $-2.192^{\star}$ & & & 5.055 & 1.947 & & & 3.226 & 1.056 & & \\
\hline & $\begin{array}{l}\text { PMPU x } \\
\text { INS_L }\end{array}$ & -0.638 & $-1.972^{*}$ & & & 0.429 & 1.360 & & & 0.240 & 0.646 & & \\
\hline \multirow[t]{3}{*}{5} & PMPU & 0.317 & $8.334^{\star \star}$ & 0.002 & 0.731 & 0.341 & 9.245 & 0.007 & 2.278 & 0.364 & $8.438^{\star \star}$ & 0.002 & 0.539 \\
\hline & IFGtriang_L & 0.398 & 0.134 & & & -0.276 & -0.096 & & & 1.482 & 0.438 & & \\
\hline & $\begin{array}{l}\text { PMPU × } \\
\text { IFGtriang_L }\end{array}$ & -0.350 & -0.855 & & & -0.597 & -1.509 & & & -0.341 & -0.734 & & \\
\hline
\end{tabular}

Note. ${ }^{*} \mathrm{P}<0.05, * * P<0.01$

\section{Discussion}

To the best of our knowledge, this is the first study to detect the moderate effect of PA and PA-related neural structure on the relationship between PMPU and emotional symptoms in college students. Our results had indicated that increasing PA could reduce the association between PMPU and emotional symptoms, and greater GMV of left INS which correlated with PA could also reduce the relationship between PMPU and depressive symptom. The results support our hypothesis that the relationship between PMPU and emotional symptoms could moderate by PA and GMV of which correlated with PA in college students.

Several studies focused the role of psychology factors on the relationship between PMPU and emotional symptoms. For example, Elhai et al (25) recruited 1034 undergraduate students and used structural equation model demonstrated that fear of missing out significantly mediated relations between problematic smartphone use and anxiety. Our previous study $(26,27)$ found that poor sleep quality may increasing the risk of emotional symptoms in PMPU students than Non-PMPU students.

There are growing more and more evidence suggests brain structural abnormality involving in PMPU and mental disorders. For PMPU, a recent study by Horvath et al (28) has found smartphone addiction group had lower GMV in left anterior insula, inferior temporal and parahippocampal cortex than health controls. Moreover, the study report smaller GMV in right lateral orbitofrontal cortex in problematic smartphone users than controls (29). Another study also takes the VBM method and find that decreased GMV of right superior frontal gyrus, right inferior frontal gyrus and bilateral thalamus in mobile phone dependence group (30). For mental disorders, a meta-analysis which included 41 studies has reported GMV difference in major depression compared to healthy controls including insula and anterior superior temporal gyrus (31). Another meta-analysis conducted of structural findings across multiple mental disorders including schizophrenia, bipolar disorder, depression, addiction, obsessive compulsive disorder and anxiety, it identified a concordance of GMV loss across in anterior insula and dorsal anterior cingulate (32). Therefore, disruption in neuro-structure may contribute to the relationship between PMPU and emotional symptoms.

It is well documented that PA has a key role in the recovery of stroke patients of movement through brain plasticity (33). For healthy participants, several cross-sectional studies have also indicated that exercise are associated with greater volume in hippocampus (34) and anterior cingulate 
cortex (35). It has also suggested exercise interventions can induce increases volume of prefrontal and anterior cingulate cortex from randomized controlled trials (36). In addition, recent systematic reviews have shown that exercise can increase the volumes in hippocampus and several cortical regions $(34,37)$. Again, the available data has supported that self-reported PA are positively related with GMV and most consistently with frontal cortex and medial temporal lobe, so do our results- FFG, PCUN, INS and IFGtriang.

Nevertheless, the studies are limited to detect the moderating effect of PA and PA-related neural structure on the relationship between PMPU and emotional symptoms in college students. Our findings show that high level of PA can reduce the relationship between PMPU and emotional symptoms, and greater GMV of left INS which correlated with PA have same protect role in the relationship between PMPU and depressive symptom. In the context of the literature, the present study expands the evidence of some neuroplastic mechanisms on PA moderated the relation between PMPU and emotional symptoms.

The insular cortex is well known for important part of 'salience' network and functions involved in interoception, autonomic control, emotional guidance of social behavior and perceptual self-awareness (38). It has been supported that insular cortex is strongly correlated with addictive behaviors, due to addictive behaviors involves in decision process such as choosing immediate rewards that always associated with physiological states that cause strong interoceptive signals (39). On the other hand, comparative quantitative meta-analysis had been performed to find a common core of areas-dorsal anterior cingulate cortex and insula are affected across most mental disorders (40). Thus, the insula is may be a key node that links the addictive behaviors and mental health.

Key strengths of the present study are neuroimaging-based and relative-large sample size. Moreover, the relatively complete information collected and power to control multiple confounding factors. However, several limitations should be acknowledged. First, this is a cross-sectional design study and limiting inferences on directionality to any of the associated factors, although we assume the direction is behavior to emotional symptoms. Second, self-reported measurements will lead to recall bias, and more objective measures should be taken in the future, such as accelerometer for PA. Lastly, it also remains some possible residual confounding factors (e.g. environmental factors), the results were not substantially changed after controlling some potential confounders.

\section{Conclusions}

This study demonstrates that high levels of PA can reducing the association between PMPU and emotional symptoms, and further find the GMV of left INS which correlated with PA may play a key role on the relationship between PMPU and depressive symptom. Future work should take longitudinal design to explore the potential protective factor of PA on the relation between PMPU and emotional symptoms and detect the neural basis of PA's moderate effects on such a relation.

\section{Abbreviations}

PMPU: problematic mobile phone use, PA: physical activity, GMV: gray matter volume, FFG: fusiform gyrus, PCUN: precuneus, INS: insula, IFGtriang: triangular part of inferior frontal gyrus

\section{Declarations}

\section{Acknowledgements:}

We sincerely thank all the adolescents and their schools for kind participation, as well as staffs of the Ping An Healthcare Diagnostics Center.

\section{Author contributions:}

T.F. design the study. Z.L., W.X., T.S., X.H., X.Y., L.T and Y.Y. performed the survey research. Z.Q, H.X, and Z.S. conducted MRI and checked the MRI data. Z.L., W.X., T.S. and X.H. analyzed the data. Z.L. draft the manuscript. Finally, all authors read and approval the final manuscript.

\section{Funding:}

This study was supported by National Natural Science Foundation of China (81773455, 81803257), Scientific Research of BSKY from Anhui Medical University (XJ201824).

\section{Availability of data and material:}


The data that support the findings of this study are available from Anhui Medical University but restrictions apply to the availability of these data, which were used under license for the current study, and so are not publicly available. Data are however available from the authors upon reasonable request and with permission of Anhui Medical University.

\section{Ethics approval and consent to participate:}

The study approved by Ethics Committee of Anhui Medical University and all participants were written informed consent.

\section{Consent for publication:}

Not applicable.

\section{Competing interests:}

The authors declare that they have no competing interests.

\section{References}

1. CNNIC. The 44th China Statistical Report on Internet Development. 2019 [Available from: http://www.cnnic.net.cn/hlwfzyj/hlwxzbg/.

2. Derevensky JL, Hayman V, Lynette G. Behavioral Addictions: Excessive Gambling, Gaming, Internet, and Smartphone Use Among Children and Adolescents. Pediatric clinics of North America. 2019;66(6):1163-82.

3. Billieux J, Philippot P, Schmid C, Maurage P, De Mol J, Van der Linden M. Is Dysfunctional Use of the Mobile Phone a Behavioural Addiction? Confronting Symptom-Based Versus Process-Based Approaches. Clinical psychology \& psychotherapy. 2015;22(5):460-8.

4. Elhai JD, Levine JC, Hall BJ. The relationship between anxiety symptom severity and problematic smartphone use: A review of the literature and conceptual frameworks. Journal of anxiety disorders. 2019;62:45-52.

5. De-Sola Gutierrez J, Rodriguez de Fonseca F, Rubio G. Cell-Phone Addiction: A Review. Frontiers in psychiatry. 2016;7:175.

6. Ng KC, Wu LH, Lam HY, Lam LK, Nip PY, Ng CM, et al. The relationships between mobile phone use and depressive symptoms, bodily pain, and daytime sleepiness in Hong Kong secondary school students. Addictive behaviors. 2020;101:105975.

7. Lissak G. Adverse physiological and psychological effects of screen time on children and adolescents: Literature review and case study. Environmental research. 2018;164:149-57.

8. Demirci K, Akgonul M, Akpinar A. Relationship of smartphone use severity with sleep quality, depression, and anxiety in university students. Journal of behavioral addictions. 2015;4(2):85-92.

9. Lapierre MA, Zhao P, Custer BE. Short-Term Longitudinal Relationships Between Smartphone Use/Dependency and Psychological Well-Being Among Late Adolescents. The Journal of adolescent health : official publication of the Society for Adolescent Medicine. 2019;65(5):607-12.

10. Park SY, Yang S, Shin CS, Jang H, Park SY. Long-Term Symptoms of Mobile Phone Use on Mobile Phone Addiction and Depression Among Korean Adolescents. International journal of environmental research and public health. 2019;16(19).

11. Visnjic A, Velickovic V, Sokolovic D, Stankovic M, Mijatovic K, Stojanovic M, et al. Relationship between the Manner of Mobile Phone Use and Depression, Anxiety, and Stress in University Students. International journal of environmental research and public health. 2018;15(4).

12. Grant JE, Lust K, Chamberlain SR. Problematic smartphone use associated with greater alcohol consumption, mental health issues, poorer academic performance, and impulsivity. Journal of behavioral addictions. 2019;8(2):335-42.

13. Elhai JD, Dvorak RD, Levine JC, Hall BJ. Problematic smartphone use: A conceptual overview and systematic review of relations with anxiety and depression psychopathology. Journal of affective disorders. 2017;207:251-9.

14. Zschucke E, Gaudlitz K, Strohle A. Exercise and physical activity in mental disorders: clinical and experimental evidence. Journal of preventive medicine and public health = Yebang Uihakhoe chi. 2013;46 Suppl 1:S12-21.

15. Chekroud SR, Gueorguieva R, Zheutlin AB, Paulus M, Krumholz HM, Krystal JH, et al. Association between physical exercise and mental health in 1.2 million individuals in the USA between 2011 and 2015: a cross-sectional study. The lancet Psychiatry. 2018;5(9):739-46.

16. Harvey SB, Overland S, Hatch SL, Wessely S, Mykletun A, Hotopf M. Exercise and the Prevention of Depression: Results of the HUNT Cohort Study. The American journal of psychiatry. 2018;175(1):28-36.

17. Schuch FB, Vancampfort D, Richards J, Rosenbaum S, Ward PB, Stubbs B. Exercise as a treatment for depression: A meta-analysis adjusting for publication bias. Journal of psychiatric research. 2016;77:42-51.

18. Rosano C, Guralnik J, Pahor M, Glynn NW, Newman AB, Ibrahim TS, et al. Hippocampal Response to a 24-Month Physical Activity Intervention in Sedentary Older Adults. The American journal of geriatric psychiatry : official journal of the American Association for Geriatric Psychiatry. 2017;25(3):209-17. 
19. Thomas AG, Dennis A, Rawlings NB, Stagg CJ, Matthews L, Morris M, et al. Multi-modal characterization of rapid anterior hippocampal volume increase associated with aerobic exercise. Neurolmage. 2016;131:162-70.

20. Tao S, Fu J, Wang H, Hao J, Tao F. The development of Self-rating Questionnaire for Adolescent Problematic Mobile Phone Use and the psychometric evaluation in undergraduates. Chinese Journal of School Health. 2013;34(1):26-9.

21. Macfarlane DJ, Lee CC, Ho EY, Chan KL, Chan DT. Reliability and validity of the Chinese version of IPAQ (short, last 7 days). Journal of science and medicine in sport. 2007;10(1):45-51.

22. Wang K, Shi HS, Geng FL, Zou LQ, Tan SP, Wang Y, et al. Cross-cultural validation of the Depression Anxiety Stress Scale-21 in China. Psychological assessment. 2016;28(5):e88-e100.

23. Aiken LS, West SG, Reno RR. Multiple regression : testing and interpreting interactions. Newbury Park, Calif.: Sage Publications; 1991. xi, 212 p. p.

24. Sanchez-Martinez M, Otero A. Factors associated with cell phone use in adolescents in the community of Madrid (Spain). Cyberpsychology \& behavior : the impact of the Internet, multimedia and virtual reality on behavior and society. 2009;12(2):131-7.

25. Elhai JD, Yang H, Fang J, Bai X, Hall BJ. Depression and anxiety symptoms are related to problematic smartphone use severity in Chinese young adults: Fear of missing out as a mediator. Addictive behaviors. 2020;101:105962.

26. Tao S, Wu X, Zhang Y, Zhang S, Tong S, Tao F. Effects of Sleep Quality on the Association between Problematic Mobile Phone Use and Mental Health Symptoms in Chinese College Students. International journal of environmental research and public health. 2017;14(2).

27. Zou L, Wu X, Tao S, Xu H, Xie Y, Yang Y, et al. Mediating Effect of Sleep Quality on the Relationship Between Problematic Mobile Phone Use and Depressive Symptoms in College Students. Frontiers in psychiatry. 2019;10:822.

28. Horvath J, Mundinger C, Schmitgen MM, Wolf ND, Sambataro F, Hirjak D, et al. Structural and functional correlates of smartphone addiction. Addictive behaviors. 2020;105:106334.

29. Lee D, Namkoong K, Lee J, Lee BO, Jung YC. Lateral orbitofrontal gray matter abnormalities in subjects with problematic smartphone use. Journal of behavioral addictions. 2019;8(3):404-11.

30. Wang Y, Zou Z, Song H, Xu X, Wang H, d'Oleire Uquillas F, et al. Altered Gray Matter Volume and White Matter Integrity in College Students with Mobile Phone Dependence. Frontiers in psychology. 2016;7:597.

31. Wise T, Radua J, Via E, Cardoner N, Abe O, Adams TM, et al. Common and distinct patterns of grey-matter volume alteration in major depression and bipolar disorder: evidence from voxel-based meta-analysis. Molecular psychiatry. 2017;22(10):1455-63.

32. Goodkind M, Eickhoff SB, Oathes DJ, Jiang Y, Chang A, Jones-Hagata LB, et al. Identification of a common neurobiological substrate for mental illness. JAMA psychiatry. 2015;72(4):305-15.

33. Hara Y. Brain plasticity and rehabilitation in stroke patients. Journal of Nippon Medical School = Nippon Ika Daigaku zasshi. 2015;82(1):4-13.

34. Firth J, Stubbs B, Vancampfort D, Schuch F, Lagopoulos J, Rosenbaum S, et al. Effect of aerobic exercise on hippocampal volume in humans: A systematic review and meta-analysis. Neurolmage. 2018;166:230-8.

35. Bento-Torres J, Bento-Torres NVO, Stillman CM, Grove GA, Jr., Huang H, Uyar F, et al. Associations between cardiorespiratory fitness, physical activity, intraindividual variability in behavior, and cingulate cortex in younger adults. Journal of sport and health science. 2019;8(4):315-24.

36. Ruscheweyh R, Willemer C, Krüger K, Duning T, Warnecke T, Sommer J, et al. Physical activity and memory functions: an interventional study. Neurobiology of aging. 2011;32(7):1304-19.

37. Zheng J, Stevenson RF, Mander BA, Mnatsakanyan L, Hsu FPK, Vadera S, et al. Multiplexing of Theta and Alpha Rhythms in the AmygdalaHippocampal Circuit Supports Pattern Separation of Emotional Information. Neuron. 2019;102(4):887-98 e5.

38. Benarroch EE. Insular cortex: Functional complexity and clinical correlations. Neurology. 2019;93(21):932-8.

39. Droutman V, Read SJ, Bechara A. Revisiting the role of the insula in addiction. Trends in cognitive sciences. 2015;19(7):414-20.

40. Downar J, Blumberger DM, Daskalakis ZJ. The Neural Crossroads of Psychiatric Illness: An Emerging Target for Brain Stimulation. Trends in cognitive sciences. 2016;20(2):107-20.

\section{Figures}




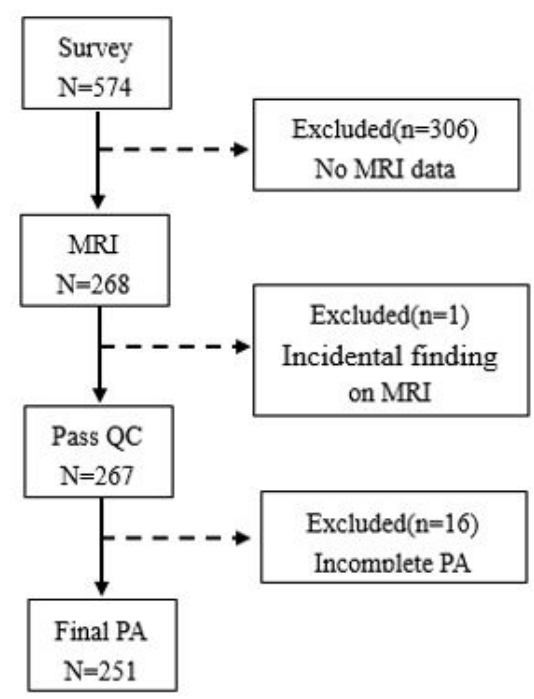

Figure 1

Flow chart of data exclusion in present study. Note. MRI: Magnetic resonance imaging, QC: quality control, PA: physical activity.

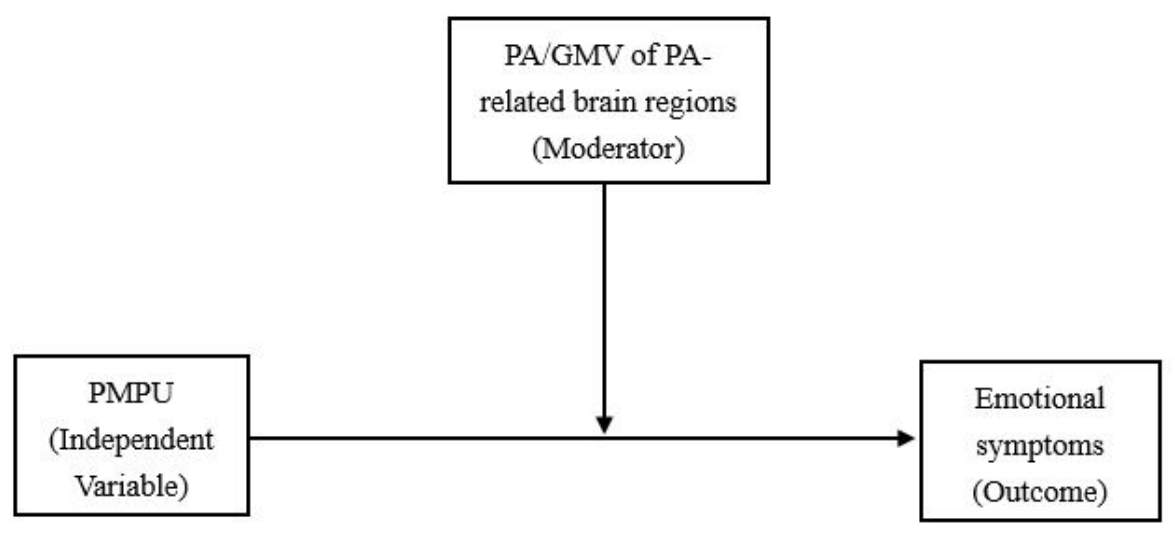

Figure 2

The proposed moderated model. Note. PMPU: problematic mobile phone use, GMV: gray matter volume, PA: physical activity. 

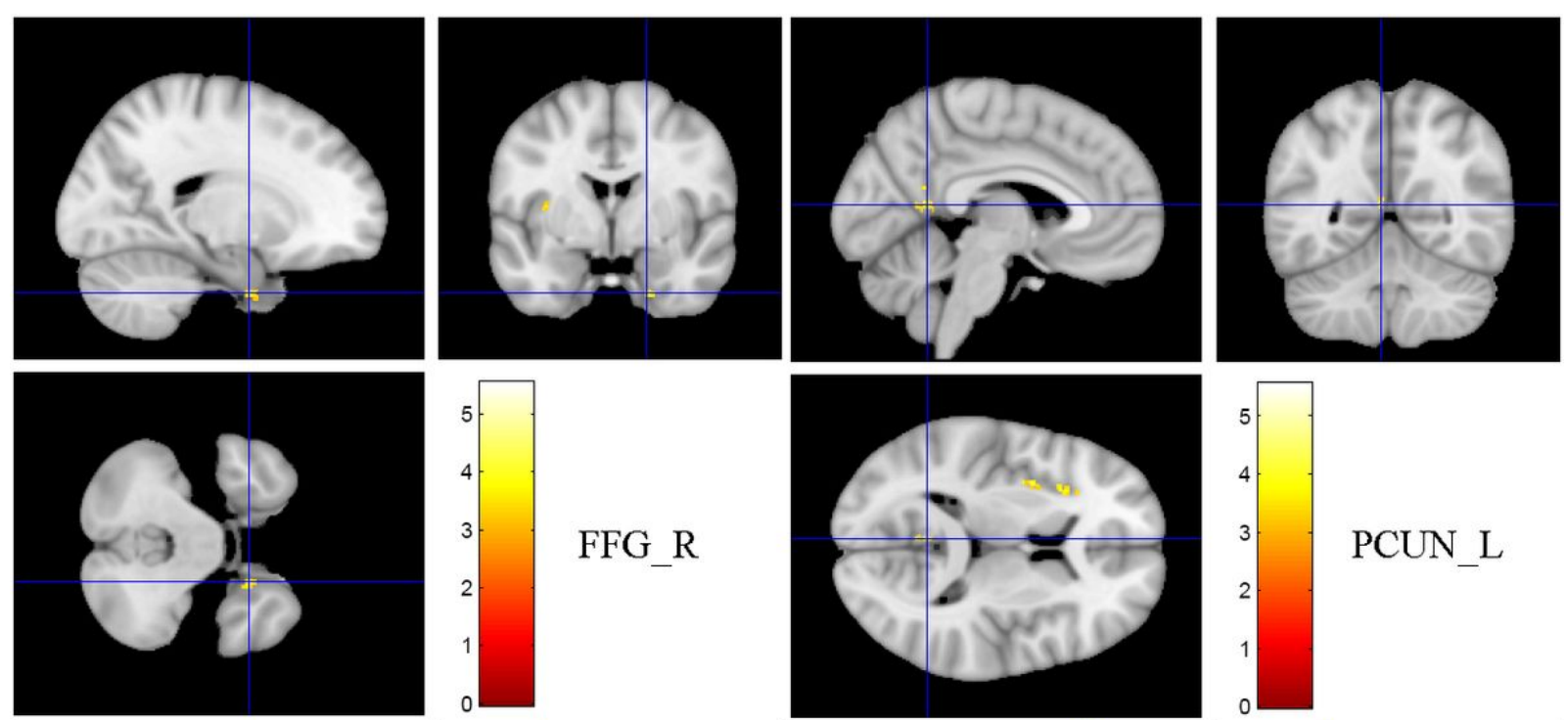

PCUN_L
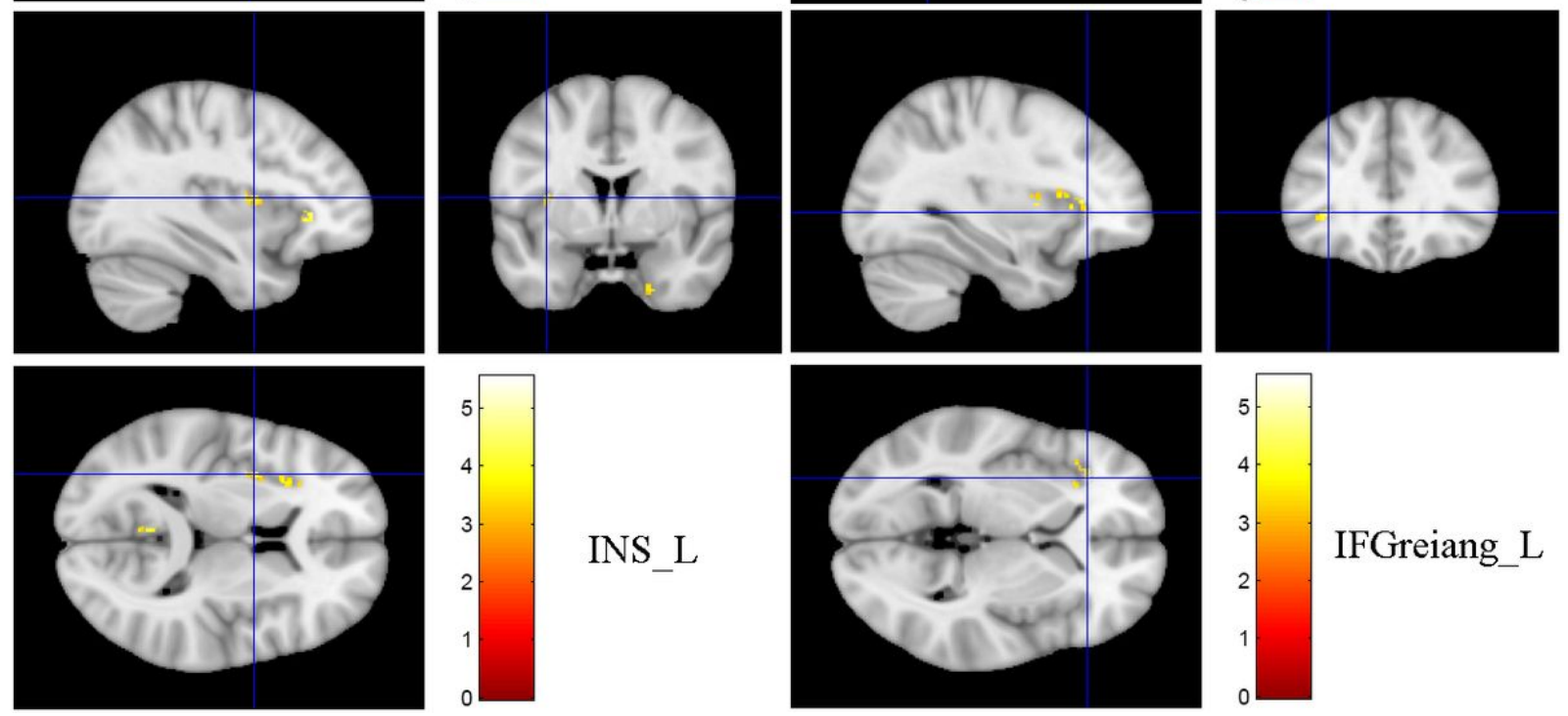

Figure 3

The red-yellow color indicates positive correlation of total PA MET and gray matter volume in the right fusiform (FFG), the left precuneus (PCUN), the left insula (INS) and the left triangular part of inferior frontal gyrus (IFGtriang). The color scale represents $t$ values. The threshold for displaying was set to $p<0.05$, FWE corrected. 

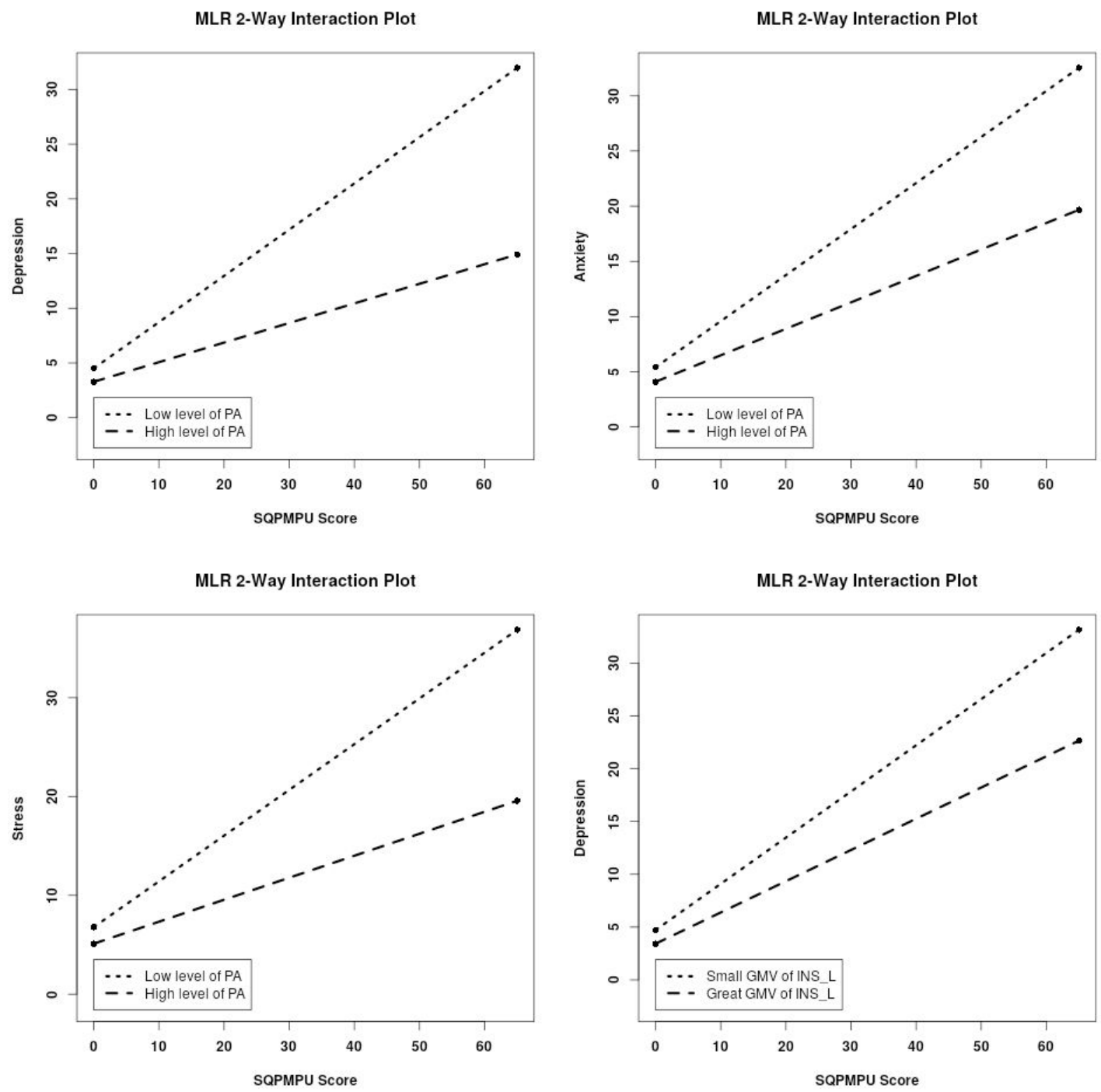

Figure 4

PA and PA-related GMV moderated the association between PMPU and emotional symptoms. Simple slopes are plotted at low (M-1SD) and high (M+1SD) PA/ GMV of left INS that correlated with PA level.

\section{Supplementary Files}

This is a list of supplementary files associated with this preprint. Click to download.

- supplyment.docx 